Traore, David Sie Chief Scientist, ORBIT david@orbitzone.space (702)445-9454 United States of America

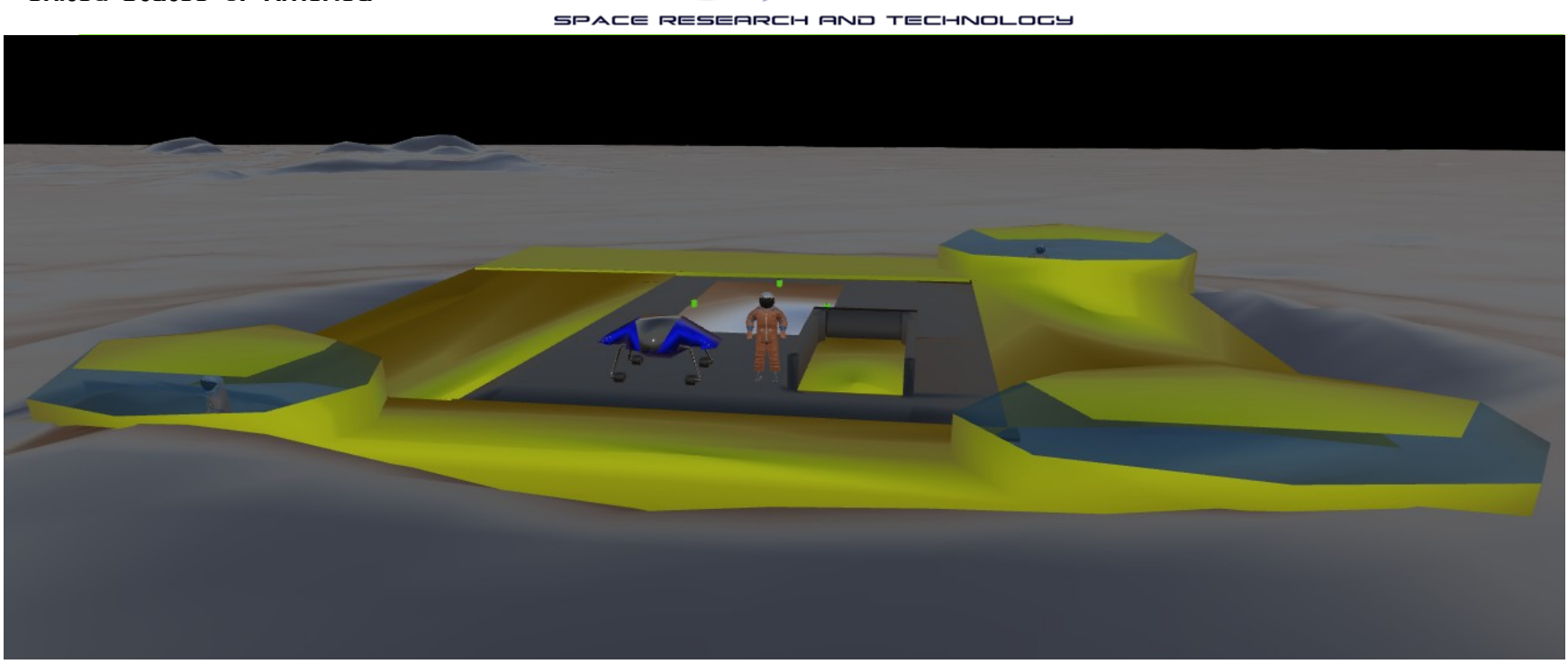

\title{
Cis-Lunar Space Neutrino Subterranean Asset
}

[Access type]: Robotics

[Year]: 2030

[Mission]: Artemis V

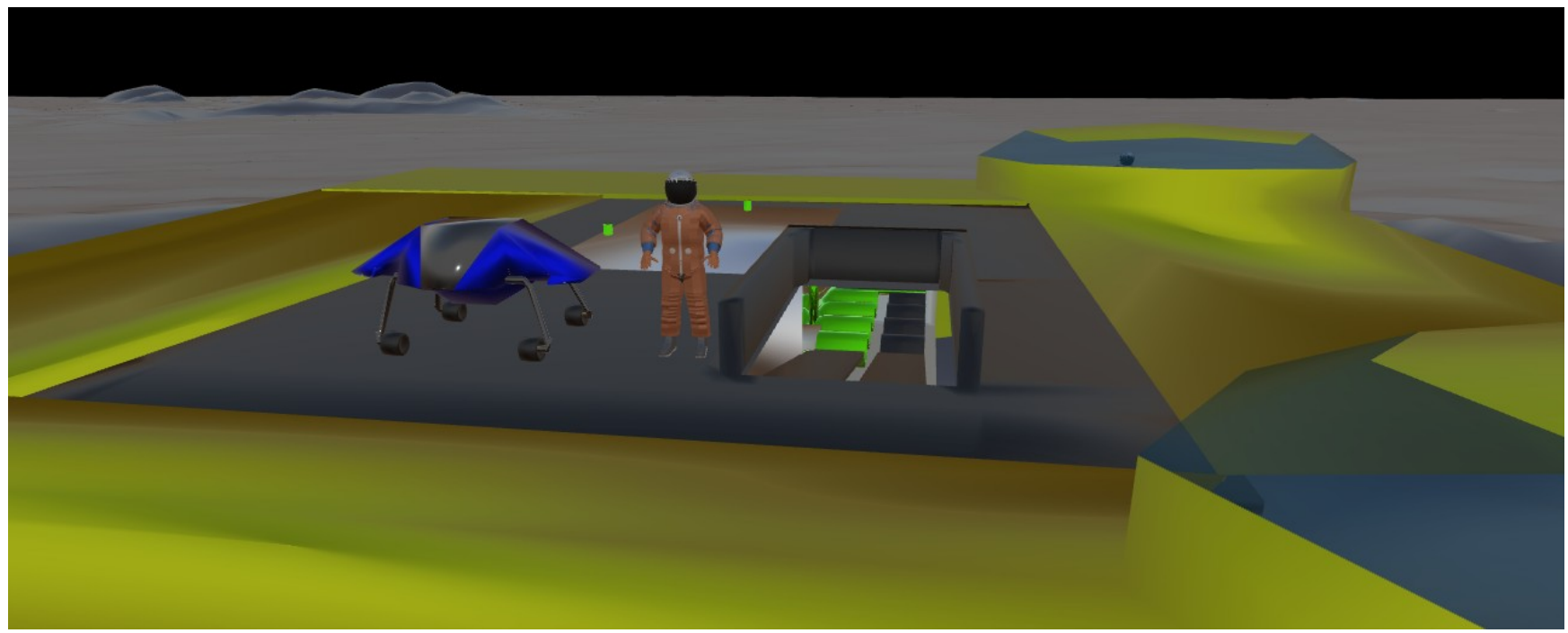

Virtual Environment

National Academies Planetary Science and Astrobiology Decadal Survey 2023-2032 


\section{Genesis}

1. In the beginning God created the Heavens and the Earth. Now the Earth was formless and empty, darkness was over the surface of the deep, and the Spirit of God was hovering over the waters. And God said: "Let there be light," and there was light. HIP 41378 is a star located at 380 light years away in the constellation of cancer, with an apparent magnitude of 8.92. It has a mass of $1.15 \mathrm{M} \odot$ and a radius of $1.4 \mathrm{R} \odot$. It's surface temperature is about $6199 \mathrm{k}$. As of today it is known to host five potential exo-planetary candidates. The system was discovered in Kepler's K2 data in 2016. Below is a plot of the K2 light curve of HIP 41378. There are several dips throughout caused by transiting planets.

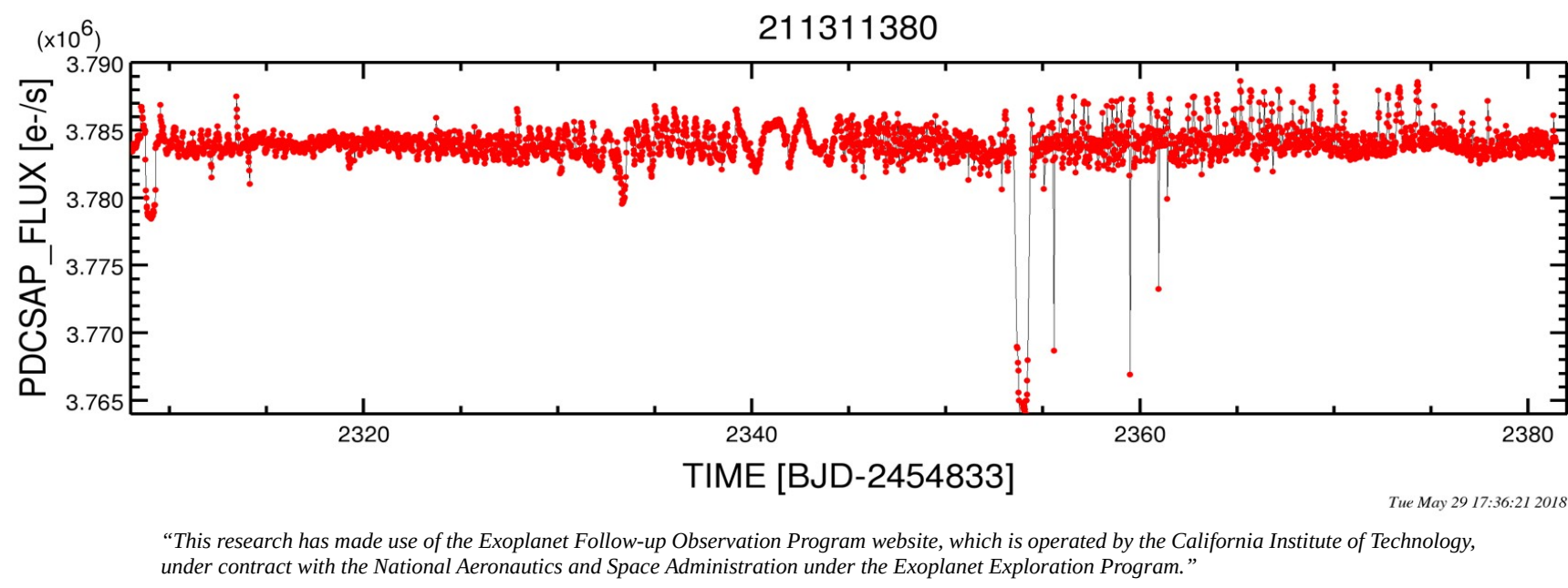

The objective is to observe this exo-system with the Cis-Lunar Space Neutrino Laboratory ( https://pcos.gsfc.nasa.gov/physpag/meetings/AAS Jan2020/CLuSN.pdf ) to generate data of its planets atmospheric composition . The system includes two sub-Neptune-sized planets: HIP 41378 c and HIP 41378 b which were both observed over multiple transits. They orbit in a nearly 2:1 resonance at periods of $31.697800000 \pm 0.004000000$ and $15.571200000 \pm 0.001200000$ days. Modeling of their transits revealed that they have radii of $2.56 \pm 0.4$ and $2.9 \pm 0.44$ Earth radii. The system also contains three larger outer-planet candidates. One Neptune-sized: HIP 41378 d (3.96 \pm 0.59 Earth radii), one sub Saturn-sized: HIP 41378 e (5.51 \pm 0.77 Earth radii), and one Jupiter-sized: HIP 41378 f (10.2 \pm 1.4 Earth radii). These planets were detected with only a single transit each, so their properties are harder to determine. Observation of their parameter suggested that their orbital periods are $56.000000000 \pm 163.000000000$ days, $131.000000000 \pm 61.000000000$ days, and $324.000000000 \pm 121.000000000$ days. The system's brightness, accessible size of its planets and rich architecture make it an excellent target for follow-up observations.

The transit depth of the outermost planet, HIP 41378 f (having a similarity with Earth's period), particularly make this candidate an ideal target for Optical \& IR spectroscopy measurements. Infrared spectroscopy of exoplanets allows molecule detection in their atmosphere. Water (H2O), methane (CH4), carbon dioxide (CO2), and carbon monoxide (CO) have been detected in two hot-Jupiter type exoplanets during primary eclipse and/or secondary eclipse events (when the planet passes either in front of or behind the stellar primary). Since past observations of exoplanet atmospheres have been primarily of shortperiod, obtaining high-resolution transit spectrum of the atmosphere of those five planets, and possibly detect and characterize other candidates in HIP 41378 could reveal Extrasolar phenomena not previously explored. 
Kepler rescue mission: In 2026, CLuS'N plans to demonstrate advancement in galactic propulsion by traveling up to $1.7 \mathrm{e}-5$ light years away from the Sun at a record breaking velocity for a rendez-vous with Kepler.

\section{Cis-Lunar Space Neutrino Laboratory (CLuS’N Lab)}

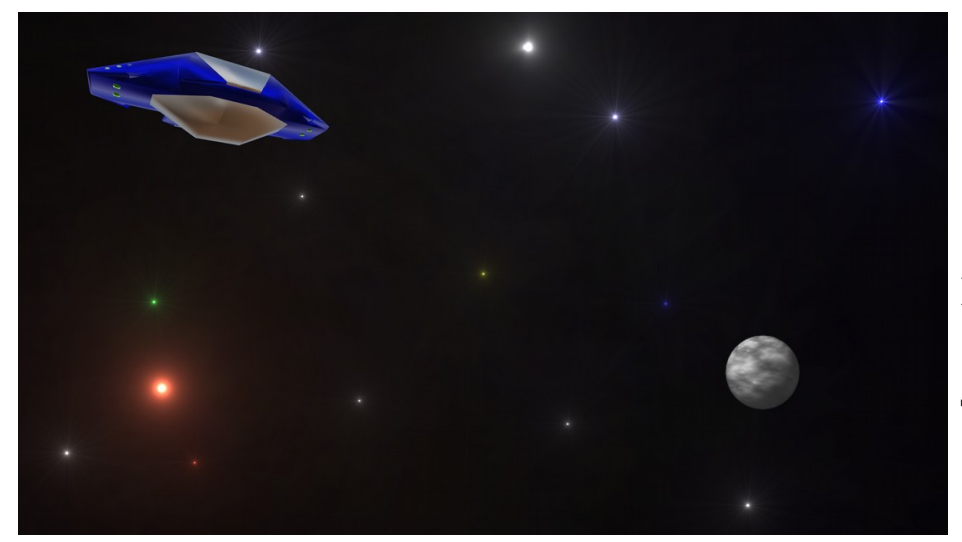

[ In space propulsion engines( $\mathrm{x} 16)$

[ Robotic Spacecraft: ESPA class

[ Manned Spacecraft (uno):

ESPA-grande class

[ Soft \& Hard X-rays, MeV gamma rays,

$\mathrm{TeV}$ gamma rays sensors.

The CLuS'N Lab is being developed to study the origins, properties, and potential applications of Multi-messenger high energy Neutrinos. Neutrinos are elementary particles traveling at nearly the speed of light believed to have no electric charge, that produce charged particles when they collide with an atom. The effort aims at establishing a sustained Neutrino research facility in orbit around the Moon. Why around the moon? Because the moon weak magnetic field will allow us to detect, track and analyze neutrinos at a faster rate from cosmic accelerators and perform In Situ Resource Utilization experiments on the Lunar surface without measurement interference from LEO satellite constellations. Neutrinos have still not been identified in connection with Gravitational Wave events and I believe that the proposed mission will address this gap. Advanced instruments, sensors, and smart materials made out of engineered nano-metallic particles supporting the harsh environment of space, creating CLuS

Neutrinos $\left(\mathbf{v}_{\mathbf{c}}\right)$ from Cis-Lunar and Lunar surface resources such as Helium-3 could for example, yield insight into future in-space propulsion technologies to study the Gravitational Wave of distant galaxies.

The National Science Foundation (NSF)'s IceCube Neutrino Observatory currently made of 5,160 sensors on 86 strings set up on a billion ton of ice equivalent to $10^{\wedge}{ }^{38}$ atoms is being upgraded to its $2^{\text {nd }}$ generation.

The CLuS'N Lab is an ESPA class spacecraft that uses NASA Gateway for communication relay purposes, with a built-in Planetary Defense application:

a Near Lunar Objects detection algorithm working in harmony with its O/IR micro-telescope.

The Moon atmosphere is known to be composed of neon, helium, and hydrogen molecules (Apollo 17). We also know that Tritium is produced when Galactic Cosmic Rays collide with hydrogen molecules, and that Helium 3 is a non radioactive daughter isotope of ${ }^{3} \mathrm{H}$ half-life (12.32 years) beta decay. The CLuS'N lab will observe the 3 molecules for the purpose of capturing in real time not just the birth of ${ }^{3} \mathrm{H}$ but also the resulting elements from the interaction of Soft \& hard, $\mathrm{MeV}$, and $\mathrm{TeV}$ gamma rays with neon and helium. It will then examine the produced particles and engage a differential analysis formula to the observed rays, that is compiling out-of-space messages from which the Neutrinos will be processed. 


\section{Nuclear Fusion Lunar Landerover (NFLL)}

The NFLL is a small robotic spacecraft (ESPA-Class, 106cmx116cmx96 cm, < 180kg) intended to land on the Moon and perform nuclear physics experiments with In-Situ Resources. It has onboard the ESIRA (Extraterrestrial Scientific Investigations Robotic Arm) instrument. The science objectives of the ESIRA is to detect, analyze and quantify the following raw materials using sCMOS technology with integrated Laser Induced Breakdown Spectroscopy (LIBS), propose nuclear fusion experiments based on findings to be carried inside the NFLL, and provide the raw materials to the NFLL :

-Iron, -Gold, -Titanium, -Uranium, -Cobalt, -Palladium, -Platinum, -Tungsten, -Water, Helium-3

The science objectives of the NFLL are to:

a)Process Deuterium and Helium-3 to produce helium and proton for moon based spacecraft.

b)Process the ESIRA newly proposed nuclear fusion experiments based on findings.

c)Process water molecules to sustain life as we know it.

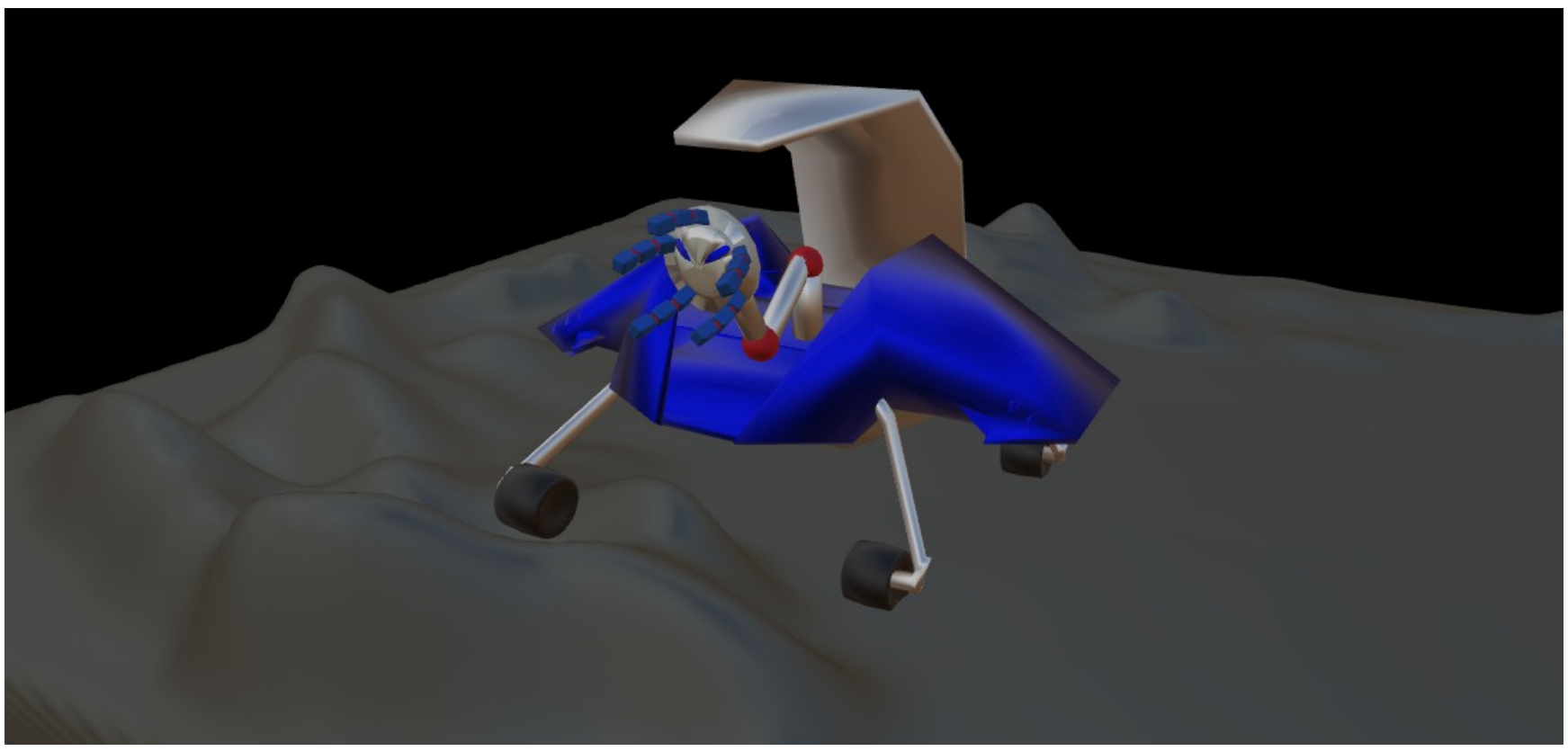

[Nuclear Fusion Lunar Landerover]: Fully autonomous

The concept is to launch the CluS'N Lab along side the NFLL. They will fly in formation to the lunar orbit. The NFLL will land on the Moon while CLuS'N remains in orbit.

4. NeCo (Neural Correlates of Computerized system interaction in deep space missions)

The challenge: "Demonstrate advance human-computer interface technologies ability to dramatically improve and naturalize interaction between a human operator and a computerized system" in deep space missions. The idea is to design a space ready quantum processing unit (NeCo) that can read the astronaut thoughts and relay the data wirelessly to the computerized system. We will measure both fNIRS (functional Near Infrared Spectroscopy) to monitor hemodynamic responses associated with neuron behavior) and EEG (electrical activity) simultaneously. We will record signals coming from the higher mental functions, eye 
movement, voluntary motor function, sensory, language comprehension, somatosensory association, and vision on a subject performing different computerized tasks in micro-gravity, EVAs, and astrophysical data analysis. The signals will be processed and coded on Neco. Upon activation, the chip will listen to incoming signals from the brain and compare the data with the preloaded models. In doing so, it will predict what the subject intends to accomplish and convert the signals to instructions. The subject needs not to remember exactly how to perform a particular task due to the fact that he or she has already done it and its been coded on the chip. This method allocates memory for the astronaut to focus on more delicate tasks and unforeseen events in the case of a Moon To Mars mission.

The subjects: "1 Female (the CO-I), 1 Male (the PI)":

- The first phase, Artemis I, 2020-2022, consists of monitoring the subjects regional tissue oxygenation and electrical activity of the brain using a portable fNIRS/EEG headset on parabolic flights at $\mathrm{G}$ levels between 0 and 1, including hyper-gravity and designing the NeCo chip. Neuroscience of learning will be recorded while the subjects learn formation flying on NASA T38 training jets, tail numbers of N912NA and N914NA located at the Johnson Space Center. Protocols of the neural circuitry will be embedded into NeCo.

- The second phase, Artemis II, 2022-2024, consist of a 30 day mission to the ISS followed by Lunar orbit missions. The subjects will monitor and record brain activities during flights and EVA. They will further develop NeCo from data collected, develop a robotic system for ISRU, and demonstrate the interaction between human and Robotic ISRU systems at the Black Point Lava Flow in Arizona and on the Nevada desert.

- $\quad$ The Third phase, Artemis III, 2024-2026, is to demonstrate human-Robotic ISRU system interaction on the lunar surface. The subjects and the NFLL will merge thoughts to extract $\mathrm{He}^{3}$ from the lunar surface. The subjects will spend a total of 7 consecutive days (number of days subject to change based on the subjects Galactic Cosmic Rays Neural Clock) on the lunar surface. The NFLL will operate no less than 2 years.

5. Artemis IV, 2026-2028

On the Lunar surface, the NFLL will be age dating ${ }^{3} \mathrm{H}$ and $\mathrm{He}^{3}$ for a comparative analysis against molecules in the upper atmosphere to confirm that $\mathrm{He}^{3}$ found on the moon is either the result or not of ${ }^{3} \mathrm{H}$ decay, including sources not previously explored.

6. Artemis V, 2028-2030

is a Planetary Protected Martian rock sample return to the Moon by a Nuclear Fusion Martian Spacecraft then on to Gateway to be delivered to Earth by the subjects onboard NASA Orion spacecraft.

7. Human-Robotics Mars Exploration (2030-2032)

The subjects and a Nuclear Fusion Martian Spacecraft will demonstrate $\mathbf{v}_{\mathbf{c}}$, energy technology and deploy a Real-time Deep-space Astro-science (RDA) communication system on the red planet.

References:

https://civspace.jhuapl.edu/News-and-Events/events/Access2Space

Lunar Surface Science Mobility Systems

PRISM, Science Mission Directorate, NASA 